\title{
La trajectoire de soins au sein d'équipes interprofessionnelles en oncologie: perspectives de patients et de proches
}

\author{
par Karine Bilodeau, Sylvie Dubois et Jacinthe Pepin
}

\section{ABRÉGÉ}

Pour accompagner le patient diagnostiqué d'un cancer dans la trajectoire de soins, des équipes de professionnels ont été créées intégrant une pratique interprofessionnelle centrée sur le patient (ICP). Le but de cet article est de présenter des résultats d'une étude de cas documentant la pratique ICP d'équipes au cours de la trajectoire de soins en oncologie. Des observations et des entrevues auprès de patients et de proches ont été réalisées. Les résultats suggèrent que la pratique ICP des équipes est variable, mais optimale en début de traitements ou lors de la récidive du cancer. Cependant, les patients peuvent faire l'expérience d'un bris dans la continuité des soins et de transitions plus difficiles entre les périodes de la trajectoire en oncologie (diagnostic, traitement, suivi).

Mots-clés : équipe multidisciplinaire, trajectoire de soins, cancer, soins centrés sur le patient, perspective de patient

\section{INTRODUCTION}

T es politiques canadiennes encouragent l'accessibilité aux ـ-services de santé et l'importance d'offrir aux personnes diagnostiquées d'un cancer des soins intégrés tout au long de la trajectoire de soins (Partenariat canadien contre le cancer, 20I3). Toutefois, cette clientèle rapporte faire face à des soins fragmentés et inaccessibles (Thorne \& Truant, 20I0). A cet effet, les équipes interprofessionnelles en oncologie représentent une solution pour faciliter le parcours du patient tout au long de sa trajectoire (Direction de lutte contre le cancer [DLCC], 2007). Ces équipes offrent des services relatifs au dépistage, à l'investigation, au traitement et à la réadaptation,

\section{AU SUJET DES AUTEURES}

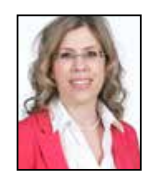

Karine Bilodeau, inf., Ph.D., CSIO(c), poursuit un stage postdoctoral au sein du programme des sciences cliniques de I'Université de Sherbrooke et est chercheure boursière au Centre de recherche de l'Hôpital Charles Lemoyne. Cette étude a été conduite dans le cadre du programme d'études doctorales en sciences infirmières de l'Université de Montréal. Courriel : karine. bilodeau2@usherbrooke.ca

Veuillez adresser toute correspondance à cette auteure

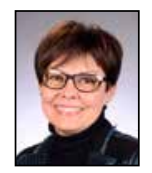

Sylvie Dubois, inf., Ph.D., est directrice des soins infirmiers du Centre hospitalier de l'Université de Montréal (CHUM) et professeure associée à la Faculté des sciences infirmières de l'Université de Montréal.

Jacinthe Pepin, inf., Ph.D., est professeure titulaire à la Faculté des sciences infirmières de l'Université de Montréal et directrice du Centre innovation en formation infirmière (CIFI) de l'Université de Montréal.

DOI: $10.5737 / 236880762512329$ en plus de communiquer de l'information pertinente et d'apporter un soutien psychologique, social et spirituel (DLCC, 2007).

Considérant que les soins pour la personne atteinte de cancer peuvent être limités s'ils sont non continus (Lewin, Skea, Entwistle, Zwarenstein, \& Dick, 200I), exercer une pratique interprofessionnelle centrée sur le patient (ICP) tout au long de la trajectoire semble être une nécessité. Cette pratique permet, grâce aux diverses expertises et contributions de chacun des professionnels, de présenter à la personne atteinte de cancer une vision intégrée et non morcelée de l'ensemble de sa situation de santé (Legault, 2003). D'ailleurs, les études réalisées auprès de patients vivant avec un cancer rapportent que ces derniers apprécient une attitude d'ouverture et de respect de la part des professionnels (Kvale \& Bondevik, 2008), mais déplorent ne pas recevoir l'information qui répond à leurs besoins et ne pas participer à une prise de décision partagée (Sinfield, Baker, Agarwal, \& Tarrant, 2008). De plus, la recension des écrits de Barnet et Shaw (2013) souligne que ces patients souhaitent une attention particulière à leur santé psychologique, une diminution des temps d'attente et l'attribution d'une personne ressource pour répondre à leurs questions, par exemple, une infirmière pivot. Selon ces auteurs, le plus grand défi des équipes interprofessionnelles est de maintenir les soins, du diagnostic à la fin de vie, tout en s'assurant qu'elles demeurent centrées sur le patient. Malgré l'importance d'exercer une pratique ICP, peu d'études, à notre connaissance ont documenté cette pratique selon la trajectoire de soins en oncologie et les perspectives de patients/proches.

Dans le cadre du projet de recherche doctorale de la première auteure, une étude qualitative de cas multiples a été réalisée. Cette étude avait pour but de décrire la pratique ICP au cours de la trajectoire de soins en oncologie. Létude devait répondre à la question de recherche suivante: comment les équipes exercent-elles la pratique ICP au cours de la trajectoire de soins? Pour répondre à cette question, il était alors important de décrire la trajectoire de soins en oncologie et de faire état de l'intensité du déploiement de cette pratique auprès du patient et de ses proches à chacune des phases. Le présent article a pour but de présenter les résultats de recherche qui documentent exclusivement la pratique ICP d'équipes au cours de la trajectoire de soins en oncologie notamment selon l'expérience des patients et de leurs proches.

\section{La pratique ICP}

Pour soutenir la description de la pratique ICP, une adaptation du Person centred nursing framework (PCNF) de McCormack et McCance (2010) par l'inclusion de 
l'interprofessionnalité, telle que définie par Couturier et al. (2008), a été utilisée comme cadre de référence. L'adaptation du PCNF explique que, lors d'une pratique ICP, l'équipe travaille avec les croyances et les valeurs du patient, s'engage, est empathique, partage la prise de décision avec le patient et offre des soins holistiques (Bilodeau, Dubois, \& Pepin, 2013). Cette pratique se réalise alors dans un espace d'échanges communs entre les professionnels et avec les patients et les proches.

\section{La trajectoire de soins en oncologie}

La trajectoire de soins en oncologie est considérée dans cette étude selon quatre périodes : l'investigation, le diagnostic, les traitements et le suivi ( Direction de la lutte contre le cancer 20II). La période d'investigation est caractérisée par l'apparition des symptômes du cancer et l'attente des résultats des tests de dépistage et d'investigation. La période du diagnostic représente l'annonce d'un diagnostic de cancer et l'attente de traitements. Par la suite, la période des traitements se distingue par le début de ceux-ci ou par leur modification si le cancer récidive. Enfin, le suivi représente la fin des traitements et la transition vers la survivance où un suivi médical et des examens de surveillance devront être assurés.

\section{MÉTHODE}

Une étude de cas multiples (Stake, 2008) a été réalisée auprès de deux équipes interprofessionnelles en oncologie afin de décrire la pratique ICP. Ce type d'étude permet d'offrir une description riche, complexe et holiste d'un phénomène. Pour cette recherche, le phénomène à étudier était la pratique ICP tout au long de la trajectoire de soins en oncologie. Les cas étaient deux équipes interprofessionnelles d'un centre hospitalier universitaire (CHU) d'une région urbaine au Québec, dédiées à un type de cancer distinct qui touche autant les hommes que les femmes. Suite à l'obtention du certificat d'éthique du milieu clinique, les équipes ont été sélectionnées avec l'aide de gestionnaires en oncologie. Un échantillonnage intentionnel (Patton, 2002) a été utilisé pour cibler les participants. Les critères d'inclusion étaient de s'exprimer en français et d'être intéressé à participer à l'étude. Quant aux patients, ils devaient également être diagnostiqués d'un cancer; se situer à une des périodes de la trajectoire de soins se déroulant en centre hospitalier soit le diagnostic, les traitements incluant la récidive et le suivi; être traités au CHU de l'étude et avoir une condition physique et mentale évaluée comme étant stable par les professionnels référents. Aucun critère précisant la stabilité physique et mentale n'a été offert aux professionnels qui évaluaient le potentiel des patients à participer à l'étude. Quant aux proches, ils devaient accompagner le patient et être âgés de plus de 18 ans. Des professionnels ont collaboré à recruter des patients et ce sont les patients qui ont recruté les proches.

Les résultats de huit entrevues semi-dirigées avec des patients $(n=5)$ et parfois accompagné d'un proche $(n=3)$ ainsi que de l'observation avec ces patients/proches sont rapportés pour décrire la pratique ICP d'équipes au cours de la trajectoire de soins. Les questions d'entrevue portaient sur l'expérience des participants au sein d'une équipe interprofessionnelle et sur leurs perceptions d'une pratique ICP. L'observation, réalisée par la première auteure, consistait en l'accompagnement des patients recrutés lors de différentes activités au sein de l'équipe (ex., rendez-vous, traitements). Le but de l'observation était de décrire comment les patients vivaient l'expérience de la pratique ICP. Au total, 57,6 heures d'observation ont été réalisées auprès des deux équipes. Le tableau I présente les caractéristiques de l'échantillon des patients et des proches (n=II).I La majorité des participants étaient d'origine caucasienne $(n=9)$. Des noms fictifs ont été attribués aux participants.

Conforme à Stake (I995), les données transcrites (entrevues et observations) ont fait l'objet d'une analyse de contenu consistant en un processus itératif comprenant les activités suivantes : la condensation, la présentation des données et l'élaboration et la vérification des conclusions (Miles \& Huberman, 2003). De plus, les cas ont été contrastés pour en décrire les particularités. Le logiciel QDA Miner v.4.O.II a été utilisé pour la gestion des données qualitatives. Les critères de rigueur scientifique retenus étaient la crédibilité (caractère authentique

I. L'échantillon complet de l'étude doctorale était composé de 8 patients, 3 proches, I 8 professionnels et 2 gestionnaires $(\mathrm{N}=3 \mathrm{I})$.

\begin{tabular}{|c|c|c|c|c|c|c|c|}
\hline \multicolumn{4}{|l|}{ CAS: Équipe $A(n=5)$} & \multicolumn{4}{|l|}{ CAS: Équipe B $(n=6)$} \\
\hline Période de la trajectoire & Participant & Sexe & Âge & Période de la trajectoire & Participant & Sexe & Âge \\
\hline \multirow[t]{2}{*}{ Diagnostic } & \multirow[t]{2}{*}{ Vladimir } & \multirow[t]{2}{*}{$M$} & \multirow[t]{2}{*}{74} & \multirow[t]{2}{*}{ Diagnostic } & Paul & $M$ & 61 \\
\hline & & & & & Suzanne* & $F$ & 57 \\
\hline Traitement & Maria & $\mathrm{F}$ & 70 & Traitement & Cathie & $F$ & 23 \\
\hline \multirow[t]{2}{*}{ Récidive } & Diane & $\mathrm{F}$ & 56 & \multirow[t]{2}{*}{ Récidive } & \multirow[t]{2}{*}{ Janice } & \multirow[t]{2}{*}{$F$} & \multirow[t]{2}{*}{59} \\
\hline & Jenny* & $\mathrm{F}$ & 26 & & & & \\
\hline \multirow[t]{2}{*}{ Suivi } & \multirow[t]{2}{*}{ Béatrice } & \multirow[t]{2}{*}{$F$} & \multirow[t]{2}{*}{48} & \multirow[t]{2}{*}{ Suivi } & Céline & $F$ & 53 \\
\hline & & & & & Marc* & $M$ & 52 \\
\hline
\end{tabular}


et plausible de la recherche), la fiabilité (stabilité des données au cours de l'analyse), l'imputabilité procédurale (description $\mathrm{du}$ processus de recherche) et la transférabilité (potentiel de transfert des connaissances chez le lecteur) (Guba \& Lincoln, 1989). Pour répondre à ces critères, l'observation soutenue effectuée auprès des équipes, la validation interjuge et la triangulation des données des entrevues et de l'observation ont été réalisés. De plus, c'est par une relecture et la condensation des données qu'il a été possible de valider la cohérence entre les conclusions avancées. Un journal de bord a été tenu afin de documenter les impressions personnelles de la première auteure lors de la collecte de données, de colliger des notes méthodologiques et interprétatives en plus et de conserver une trace du processus de recherche. Enfin, le contexte de la pratique ICP en oncologie a été documenté de façon détaillée.

\section{RÉSULTATS}

Les résultats présentés ici portent sur l'exercice d'une pratique ICP de deux équipes à différentes périodes de la trajectoire de soins (diagnostic; traitements incluant la récidive; suivi) tel que perçu par des patients et leurs proches. Cette description est précédée par une brève présentation des caractéristiques des cas. Le schéma I présente l'implication des équipes interprofessionnelles selon la trajectoire de soins en oncologie.

\section{Caractéristiques des cas}

Équipe A. Les patients de l'équipe A étaient atteints d'un cancer où le taux de survie était de moins de cinq ans. Les principaux traitements pour ce type de cancer étaient la chirurgie et la chimiothérapie intraveineuse. Les participants devaient se rendre régulièrement à l'hôpital pour des rendez-vous et des traitements. Lors de la collecte de données, les patients ont rapporté être incommodés par les effets secondaires de la chimiothérapie (ex., nausée, asthénie). Les professionnels ${ }^{2}$ de l'équipe A se composaient de ceux qui travaillaient à l'unité de soins ainsi qu'à la clinique d'oncologie.

Équipe B. Les patients de l'équipe B étaient atteints d'un cancer où le taux de survie était de moins de deux ans. Après une biopsie ou une chirurgie, un traitement concomitant de radiothérapie et de chimiothérapie orale était offert aux patients. À la fin de ce traitement, c'est seulement un traitement de chimiothérapie qui se poursuivait. En raison de ce type de traitement, un nombre moindre de rendez-vous médicaux était nécessaire. Lors des entrevues, le discours des patients et des proches était teinté de désarroi en raison des pertes et deuils qu'ils vivaient (ex., occuper un emploi, conduire une automobile). De plus,

2. Divers professionnels de la santé composaient les équipes A et B par exemple, l'infirmière pivot, le médecin, le physiothérapeute, le nutritionniste, le psychologue, le pharmacien, le travailleur social ou l'ergothérapeute.

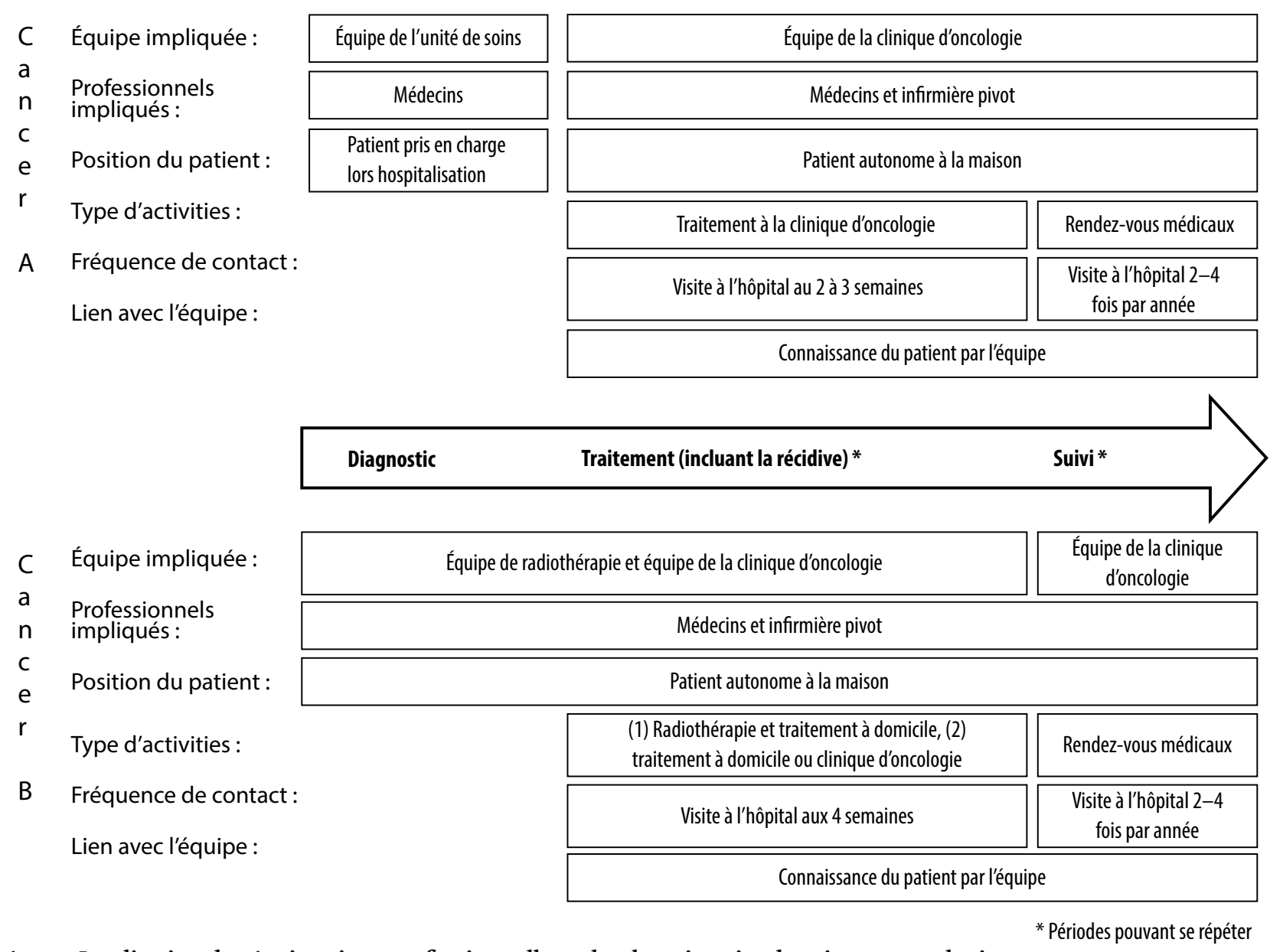

Schéma I. Implication des équipes interprofessionnelles selon la trajectoire de soins en oncologie 
parallèlement aux traitements et aux rendez-vous, les participants devaient régler, sans aide, plusieurs dossiers personnels (ex., mandat d'inaptitude, assurance-maladie). Léquipe B était composée de professionnels de l'unité de soins, de ceux de la clinique de radio-oncologie ainsi que de ceux de la clinique d'oncologie.

\section{La période du diagnostic}

Pendant la période du diagnostic, les opportunités d'interaction des participants avec les professionnels étaient différentes d'une équipe à l'autre. Les patients de l'équipe A étaient en contact avec le chirurgien où une forte relation de confiance semblait être établie depuis l'annonce du diagnostic. Béatrice explique: «Il avait ma vie entre les mains donc il fallait que je sois confiante.» Cette période se déroulait essentiellement avec les professionnels de l'unité de soins. L'observation a soulevé que les activités de l'équipe étaient axées sur les soins périopératoires. Quant à l'équipe $\mathrm{B}$, les patients étaient hospitalisés brièvement pour une biopsie ou une chirurgie. Par la suite, le diagnostic était annoncé par un des médecins lors d'une clinique interprofessionnelle où des médecins de diverses spécialités et l'infirmière pivot étaient présents et présentés aux patients.

La période du diagnostic se caractérise par le choc de l'annonce du cancer et par la nécessité de prendre une décision rapide quant aux traitements. La majorité des participants soulignaient avoir reçu beaucoup d'information. Pour l'équipe A, tous les patients admettaient avoir reçu de l'information claire et en quantité suffisante. Diane décrit: «Quand il m'a expliqué qu'il m'opérait pour ma tumeur, il m'a fait un dessin (...) parce qu'il voyait bien que je ne comprendrais rien.» Quant à l'équipe $\mathrm{B}$, des patients mentionnaient ne pas avoir été réceptifs aux nombreuses informations reçues en peu de temps par de nombreux professionnels. Ils ont mentionné qu'ils auraient apprécié une pause avant de recevoir les informations relatives aux traitements parce qu'ils n'étaient pas réceptifs. Céline partage: «Une visite de cinq, six [professionnels], tout de suite (...), je ne les ai pas écoutés (...) j'étais tellement, moi, prise par l'annonce qui m'avait été faite [diagnostic].»

Les patients devaient faire des choix rapidement quant à leurs traitements parce que les cancers (A et B) évoluaient rapidement. La majorité des patients de l'équipe $\mathrm{A}$ ont exprimé leur ambivalence quant aux choix relatifs aux traitements. Maria rapporte: «Mais je lui [médecin] ai dit aussi «À quel prix?» [accepter la chimiothérapie]. Je suis une personne très indépendante et je ne veux pas me voir comme une guenille.» Quant aux patients de l'équipe B, ils mentionnaient qu'ils n'avaient pas le choix d'accepter les traitements s'ils voulaient survivre. Cathie rapporte: «Il n'y a pas vraiment eu de décision à prendre de mon côté (...) cela a été provoqué [par les professionnels]: tu vas avoir de la chimio, tu vas faire de la radiothérapie.»

\section{La période de traitements incluant la récidive}

Lors de cette période, la majorité des participants des deux équipes ont apprécié les «sourires» et la «sensibilité» des professionnels face à leur situation. À cet effet, Béatrice explique: «Partout, j'ai toujours été reçue avec beaucoup d'amabilité et de gentillesse.» Par ailleurs, les patients n'étaient plus hospitalisés et devaient se déplacer pour rencontrer l'équipe. Ils étaient autonomes et ils prenaient leur décision. Béatrice résume:

«C'est peut-être moi qui ne suis pas dans la même position.

D'un côté, je suis alitée [unité de soins], de l'autre côté, je suis debout [clinique d'oncologie]. (...) J'étais beaucoup plus en contrôle de ce qui se passait pour la chimiothérapie [clinique d'oncologie].» (Béatrice)

L'organisation des services différait pour chaque équipe. Pour l'équipe A, le suivi était réalisé par les professionnels de la clinique d'oncologie. L'infirmière pivot était présentée aux patients. Néanmoins, les patients ont avoué avoir gardé contact avec le chirurgien rencontré à la période du diagnostic. Trois d'entre eux ont repris rendez-vous avec ce dernier pour discuter des traitements de chimiothérapie même s'il n'était plus le médecin traitant. Quant à l'équipe $B$, les patients avaient déjà rencontré brièvement les professionnels à l'annonce du diagnostic. Comme le traitement du cancer B demandait en premier lieu un traitement concomitant de chimiothérapie orale et de radiothérapie, les services étaient d'abord offerts à la clinique de radio-oncologie. À la fin de ce traitement, les patients transféraient à la clinique d'oncologie. Ils devaient donc s'adapter à ce changement d'environnement. Suzanne explique: «Quand tu sors de la radio-oncologie, tu es comme laissée à toi-même.»

Lors de l'annonce de la récidive de cancer, il a été rapporté par les patients et observé lors de la collecte de données que les équipes mettaient tout en œuvre pour accompagner les patients. Ces derniers étaient souvent connus des équipes et cela contribuait à la personnalisation des services. Lors de l'annonce de la récidive du cancer, Diane a apprécié que l'on connaisse son histoire, qu'on lui démontre de l'empathie et qu'on lui offre une attention particulière. Elle explique: «C'est la même équipe, c'est sécurisant (...) parce que quand je suis revenue, bien je n'avais pas à réexpliquer mon cas (...) Ils étaient déjà au courant».

La plupart du temps, les patients des deux équipes rencontraient individuellement les professionnels. Par ailleurs, les fréquences de rencontres avec les équipes étaient distinctes. Comme les patients de l'équipe $\mathrm{A}$ se déplaçaient régulièrement à l'hôpital, ils pouvaient croiser des professionnels comme l'infirmière pivot ou le pharmacien. D'ailleurs, plusieurs professionnels étaient à proximité des lieux fréquentés par les patients. Jenny raconte: «Pendant la première série [de traitements de chimiothérapie], on la [infirmière pivot] voyait quand même quasiment à chaque rencontre.» Donc, les patients avaient plus d'occasions de poser des questions et d'échanger avec eux. Les patients de l'équipe B venaient seulement une fois ou deux par mois au centre hospitalier pour leur rendez-vous médical. Ils rencontraient rarement d'autres professionnels sauf l'infirmière pivot; ils pouvaient lui téléphoner pour toutes questions. Paul rapporte: «Si on avait des problèmes en général, on parlait avec l'infirmière pivot [au téléphone]».

Les patients de l'équipe A ont rapporté avoir eu le sentiment que l'équipe travaillait en «synergie» parce qu'ils avaient quelquefois des indices que les membres de ces dernières 
collaboraient. Les patients remarquaient que les professionnels rapportaient les propos des autres professionnels ou disaient ouvertement avoir discuté avec un collègue. Diane raconte:

«Tu le vois qu'il y a de la communication, parce que des fois, ils vont te parler de quelque chose que probablement ça ne les regarde pas (...) mais qu'ils sont au courant (...) Je trouve ça rassurant». (Diane)

Notons que les patients de l'équipe B soulignaient ne pas avoir remarqué de travail d'équipe, même si à l'annonce du diagnostic, les professionnels et les services disponibles leur avaient été présentés. Marc confie: «On s'est dit: ça va mal [diagnostic de cancer], mais on a une belle équipe et des services on va en avoir. Après (...) c'était nous qui fallait s'en occuper [obtenir des services].» (Marc)

Malgré la présence d'équipes interprofessionnelles, les interventions n'étaient pas pour autant plus rapides et continues auprès des patients des deux équipes. Il a aussi été observé que les professionnels pouvaient ne pas tenir compte de l'avis et de l'expérience du patient. Certains patients nous ont mentionné s'être «imposés » à l'équipe soit en participant à leurs soins (ex., tenir un journal de leurs symptômes) soit en refusant des soins. De plus, il a été rapporté et observé des bris dans la continuité de leurs soins comme un rendez-vous non organisé, un test à refaire parce que de l'information n'était pas consignée au dossier ou de l'attente pour un soin. Les patients devaient être vigilants et attentifs pour recevoir des soins adéquats. Un mémo d'observation présente cette situation:

Janice rapporte qu'en parlant au téléphone avec l'infirmière pivot, elle s'est rendue compte qu'elle n'avait pas suivi les consignes usuelles quant à la prise de sa chimiothérapie. On ne lui avait pas remis la feuille de directives. (Mémo-Janice)

Les participants de l'équipe $B$ ont rapporté ne pas avoir reçu suffisamment d'informations pendant la période des traitements. Les patients et les proches avouaient ne pas comprendre les procédures entourant les traitements. Ils avaient l'impression de «rester sur leur faim». Même si le pronostic du cancer B était sombre, les patients et les proches voulaient obtenir des informations détaillées sur le sujet. Cathie explique:

«Cela a pris du temps avant que je pose la question [quand j'allais mourir] et j'avais peur de la réponse. Mais, on ne me l'a pas dit (...) c'est quoi mon espérance de vie. Je ne sais pas si je me suis achetée quelques années (...) parce qu'on ne sait pas combien de temps je vais le faire [traitement](...) c'est quoi la suite (...) c'est quoi les signes pour que ça [cancer] revienne ou ça ne revienne pas?» (Cathie)

\section{La période du suivi}

Lors de la période du suivi, les visites à la clinique d'oncologie étaient rares (3 à 4 fois par année). Les patients des deux équipes ont rapporté avoir apprécié pouvoir contacter l'infirmière pivot pour toutes questions parce qu'ils ressentent «toujours une épée au-dessus de la tête». Cette infirmière était le point d'accès à l'équipe même si, comme l'a souligné Céline, la plupart des services étaient offerts et expliqués à la période du diagnostic et que c'était à la fin des traitements que les besoins se faisaient le plus ressentir. Elle raconte:

"J'ai dit, après 14 mois de chimio, je pense bien que j'aurais le droit à quelques séances [en psychologie] que je n'ai pas utilisées durant la première année (...) on $y$ va quand on est prêt. Ça ne sert à rien de venir ou que je vous rencontre parce que je n'aurais pas parlé [à la période du diagnostic].» (Céline)

Enfin, cette période est caractérisée par le désir d'obtenir du soutien au moment jugé opportun par les patients. La prochaine section présente la discussion des résultats.

\section{DISCUSSION}

Lors d'une pratique ICP, l'équipe de soins travaille avec les valeurs du patient, s'engage, est empathique, partage la prise de décision et offre des soins holistiques; cependant les résultats de la présente étude nous présentent des pratiques variables. À notre connaissance, peu d'études ont documenté cette pratique selon la trajectoire de soins en oncologie et les perspectives de patients/proches. Il a également été constaté que le partage d'informations entre les professionnels était inégal surtout lors de la période du diagnostic où les patients ont rapporté vouloir obtenir de l'information juste et complète, quand ils se sentent réceptifs. Les résultats montrent également que c'est lors de la période des traitements que les patients remarquent notamment le savoir-être des professionnels (leur accueil, leur amabilité), mais c'est lors d'une récidive du cancer que la pratique ICP semble être optimale. Cet élément peut être expliqué par le fait que le patient est connu de l'équipe et cette connaissance du patient semble encourager une pratique ICP.

La description de l'intensité de la pratique ICP des équipes de soins au cours de la trajectoire est une contribution originale de la présente étude. Lors de la période du diagnostic, un lien de confiance semblait davantage développé avec le médecin qu'avec les autres membres de l'équipe interprofessionnelle. C'est à la période de traitements et de la récidive que les participants ont eu le plus de contact avec l'équipe et où ils pouvaient remarquer le travail interprofessionnel. Quant à la période du suivi, les résultats soulignent le désir des patients d'avoir accès à du soutien en plus de celui de l'infirmière pivot et la difficulté à obtenir des services professionnels (ex., psychologue). Plusieurs auteurs rapportent que lors de cette période, les patients font face à de nombreuses problématiques et préoccupations (ex., fatigue, anxiété, peur de la récidive) où des soins et des services sont requis (Alfano, Ganz, Rowland, \& Hahn, 20I2; Ness et al., 20I3). Ainsi, les résultats de la présente étude suggèrent que les équipes interprofessionnelles sont plus actives auprès du patient à la période de traitements et de récidive. Par contre, les patients souhaitent un suivi interprofessionnel centré sur le patient où les professionnels ajustent leur pratique selon les particularités vécues lors de la trajectoire de soins. 
Les résultats des entrevues et des observations des patients montrent aussi que ceux-ci sont rarement témoins de la collaboration entre les membres de l'équipe. D'ailleurs, les observations ont révélé que les patients de l'équipe $B$ avaient moins de contacts avec les professionnels en raison de la chimiothérapie orale qui se déroulait à domicile. Les patients ont alors moins de possibilités d'interaction, d'évaluation et de formation auprès des membres de l'équipe (Given, Spoelstra, $\&$ Grant, 20II; Simchowitz et al., 20I0). Ceci pourrait expliquer que les patients ont exprimé ne pas remarquer le travail interprofessionnel de l'équipe $\mathrm{B}$ comparativement à l'équipe A. Le contexte de soins de chaque équipe est alors un élément important qui pourrait influencer la pratique ICP (ex., organisation des services, modalités de traitement). Ainsi, pour apprécier une pratique ICP, il semble important que les patients vivent des contacts réguliers avec les membres de l'équipe dépendamment du contexte de soins.

Les résultats ont également soulevé que la pratique ICP était variable, des bris de continuité ayant été rapportés et observés auprès des deux équipes. Comme mentionné par plusieurs auteurs (Barnet \& Shaw, 20I3; Mazor et al., 20I2), les bris de continuité quant aux délais, le partage d'information, la coordination des soins et le soutien sont des éléments influençant négativement la trajectoire de soins. Notons que depuis plusieurs années, de nombreuses initiatives gouvernementales ont été développées encourageant la mise en place d'infirmières pivots (Direction de la lutte contre le cancer, 2008) ou de navigateurs (Institute of Medicine, 20II) pour faciliter la coordination des soins et pour atténuer les fardeaux relatifs à l'expérience du patient diagnostiqué d'un cancer (ex., anxiété, problèmes financiers). Même si les patients des deux équipes étudiés avaient accès à une infirmière pivot et mentionnaient l'apprécier, il semble que ce n'était pas suffisant pour assurer la continuité des soins. Il est alors possible de croire que la continuité des soins est un indicateur de la qualité des soins lors d'une pratique ICP.

De plus, les résultats de la présente étude suggèrent que les patients ont vécu des périodes de vulnérabilité, aussi nommée «transition critique» (Clausen et al., 20I2), étant caractérisées par une diminution de contact avec certains professionnels lors de la transition d'une période de la trajectoire de soins à une autre (ex., diagnostic et début de traitements). D'une part, les patients de l'équipe $\mathrm{A}$ n'avaient pas accès à une infirmière pivot pour faciliter le transfert vers la clinique d'oncologie, mais ils se sont référés à leur médecin. D’autre part, les participants de l'équipe $B$ avaient accès à une infirmière pivot dès l'annonce du diagnostic, mais la présence de celle-ci ne semblait pas faciliter la transition de la clinique de radio-oncologie à la clinique d'oncologie. Ces résultats suggèrent que les équipes interprofessionnelles en oncologie n'offrent pas totalement des soins intégrés tout long de la trajectoire de soins. Ainsi, la transition harmonieuse entre les différentes périodes de la trajectoire de soins semble indiquer une pratique ICP optimale (Walsh et al., 20I0).
La présente étude comporte certaines limites. Notamment, mentionnons que les périodes d'observation ont été moins fréquentes pour les patients à la période du suivi en raison des visites moins fréquentes à la clinique d'oncologie. Cette situation a pu avoir pour effet d'affecter la richesse de la description de la pratique ICP pour cette période. De plus, on ne peut passer sous silence le fait que la première auteure, ayant réalisé les entrevues et les observations et qui était connue des professionnels, peut avoir influencé le déroulement de certaines interactions tant avec les patients que les professionnels. Il est aussi à préciser que les résultats de la présente étude sont en lien avec un contexte précis soit l'oncologie. L'utilisation de ces résultats doit donc faire appel à un contexte d'application similaire.

\section{Implication pour la pratique et la recherche}

Les présents résultats soulignent que l'intensité de la pratique ICP varie selon les périodes de la trajectoire de soins et le contexte de chaque équipe. Ils indiquent aussi un besoin des patients lors de la période du suivi. Il serait alors important: I) d'identifier un intervenant pivot dès le diagnostic; 2) de fournir les informations pertinentes selon la période de la trajectoire de soins; 3) d'identifier avec le patient les périodes de transitions critiques et d'y porter une attention particulière; 4) et de maximiser les fréquences de contact avec le patient et ses proches. De plus, les équipes interprofessionnelles devraient être sensibles au suivi après l'expérience du cancer et aux bris de continuité tout au long de la trajectoire de soins parce que ces bris influencent négativement l'expérience du cancer. Il est alors essentiel que les équipes optimisent leur pratique ICP.

À notre connaissance, peu de recherches ont choisi d'étudier la pratique ICP selon la perspective des patients et des proches utilisant un devis qualitatif de cas multiples. Ce devis a permis de décrire les particularités de deux équipes de soins ayant des contextes différents. D'autres recherches sont nécessaires pour documenter la pratique ICP tout au long de la trajectoire de soins pour chaque type de cancer afin d'émettre des recommandations quant à l'accompagnement des patients. Il est aussi suggéré de considérer l'observation auprès des patients et des proches comme une stratégie de recherche pertinente et complémentaire pour documenter de façon détaillée les particularités de la trajectoire de soins en oncologie.

\section{REMERCIEMENTS}

Nous reconnaissons le soutien financier de la Fondation du Centre hospitalier de l'Université de Montréal (CHUM), de la Direction des soins infirmiers du CHUM, du Ministère de l'Éducation, des Loisirs et des Sports du Québec (MELS), de la Faculté des sciences infirmières (FSI) de l'Université de Montréal, de la Faculté des études supérieures et post doctorales (FESP) de l'Université de Montréal, du Réseau de recherche en interventions en sciences infirmières du Québec (RRISIQ), de la Fondation des infirmières et infirmiers du Canada (FIIC), du Centre de Recherche du CHUM et de l'Association canadienne des infirmières en oncologie (CANO-ACIO). 


\section{RÉFÉRENCES}

Alfano, C.M., Ganz, P.A., Rowland, J.H., \& Hahn, E.E. (2OI2). Cancer survivorship and cancer rehabilitation: Revitalizing the link. Journal of Clinical Oncology, 30(9), 904-906.

Barnet, M., \& Shaw, T. (20I3). What do consumers see as important in the continuity of their care? Supportive Care in Cancer, 21(9), $2637-2642$.

Bilodeau, K., Dubois, S., \& Pepin, J. (2013). Contribution des sciences infirmières au développement des savoirs interprofessionnels. Recherche en Soins Infirmiers, II3, 43-50.

Clausen, C., Strohschein, F.J., Faremo, S., Bateman, D., Posel, N., \& Fleiszer, D.M. (2012). Developing an interprofessional care plan for an older adult woman with breast cancer: From multiple voices to a shared vision. Clinical Journal of Oncology Nursing, 16(I), EI8-25.

Couturier, Y., Gagnon, D., Carrier, S., \& Etheridge, F. (2008). The interdisciplinary condition of work in relational professions of the health and social care field: A theoretical standpoint. Journal of Interprofessional Care, 22(4), 34I-35I.

Direction de la lutte contre le cancer. (2008). Rôle de l'infirmière pivot en oncologie. Québec Ministère de la santé et des services sociaux.

Direction de la lutte contre le cancer. (2007). Orientations prioritaires 2007-2012 du programme québécois de lutte contre le cancer. Québec: MSSS du Québec.

Direction de la lutte contre le cancer. (20II). Rapport du comité d'oncologie psychosociale: Vers des soins centrés sur la personne. Québec: MSSS.

Given, B.A., Spoelstra, S.L., \& Grant, M. (20II). The challenges of oral agents as antineoplastic treatments. Seminars in Oncology Nursing, 27(2), 93-I03.

Guba, E.G., \& Lincoln, Y.S. (1989). Fourth generation evaluation. Newbury Park: Sage Publications.

Institute of Medicine. (20II). Patient-centered cancer treatment planning: Improving the quality of oncology care: Workshop summary. Washington: National Academies Press.

Kvale, K., \& Bondevik, M. (2008). What is important for patient centred care? A qualitative study about the perceptions of patients with cancer. Scandinavian Journal of Caring Sciences, 22(4), $582-589$.

Legault, G.A. (2003). Crise d'identité professionnelle et professionnalisme. Québec: Presses de l'Université du Québec.

Lewin, S., Skea, Z.C., Entwistle, V., Zwarenstein, M., \& Dick, J. (200I). Interventions for providers to promote a patient-centred approach in clinical consultations. Cochrane Database Syst Rev(4), CDoo3267.
Mazor, K.M., Roblin, D.W., Greene, S.M., Lemay, C.A., Firneno, C.L. Calvi, J., et al. (20I2). Toward patient-centered cancer care: Patient perceptions of problematic events, impact, and response. Journal of Clinical Oncology, 30(I5), I784-I790.

McCormack, B., \& McCance, T.V. (2010). Person-centred nursing: Theory and practice. Oxford: John Wiley and Sons.

Miles, M.B., \& Huberman, A.M. (2003). Analyse de données qualitatives (2e ed.). Paris: De Boeck Université.

Ness, S., Kokal, J., Fee-Schroeder, K., Novotny, P., Satele, D., \& Barton, D. (20I3). Concerns across the survivorship trajectory: Results from a survey of cancer survivors. Oncology Nursing Forum, 40(I), $35-42$.

Partenariat canadien contre le cancer. (2013). Amélioration de l'expérience globale du cancer 2013. From http://www. partnershipagainstcancer.ca/fr/priorities/cancer-journey/

Patton, M.Q. (2002). Qualitative research \& evaluation methods (3rd ed.). Thousand Oaks, Calif.: Sage Publications.

Simchowitz, B., Shiman, L., Spencer, J., Brouillard, D., Gross, A., Connor, M., et al. (2010). Perceptions and experiences of patients receiving oral chemotherapy. Clinical Journal of Oncology Nursing, 14(4), 447-453.

Sinfield, P., Baker, R., Agarwal, S., \& Tarrant, C. (2008). Patientcentred care: What are the experiences of prostate cancer patients and their partners? Patient Education and Counseling, 73(I), 9I-96.

Stake, R.E. (I995). The art of case study research. Thousand Oaks: Sage Publications.

Stake, R.E. (2008). Qualitative case studies. In N.K. Denzin \& Y.S. Lincoln (Eds.), Strategies of qualitative inquiry (3rd ed., pp. II9I49). Los Angeles: Sage Publications.

Thorne, S., \& Truant, T. (20I0). Les intervenants pivots solutionneront-ils le problème? Les soins infirmiers en oncologie en transition. Revue canadienne de soins infirmiers en oncologie, 20(3), I22-I28.

Walsh, J., Harrison, J.D., Young, J.M., Butow, P.N., Solomon, M.J., \& Masya, L. (20I0). What are the current barriers to effective cancer care coordination? A qualitative study. BMC Health Services Research, 10, 132 . 